\title{
Diagnosing Language Variations: Underlying Principles for Syntactic Assessment
}

\author{
Thomas Roeper, Ph. D. ${ }^{1}$
}

\section{ABSTRACT}

The assessment of complex aspects of children's syntactic development can be carried out in a dialect-neutral fashion. The item types proposed for this purpose encompass wh-questions, passives, and articles, and test the child's understanding of implicit grammatical relations, the rules governing syntactic movement, and discourse linking. We present the rationale for the items and the specific research supporting them, as well as some suggestions for how to help children who fail on the concepts to gain a better understanding of them.

KEYWORDS: Implicit information, discourse linking, double wh-questions, wh-barriers; definite versus indefinite articles, passive, hidden agents, exhaustive set, singleton answers, medial question

Learning Outcomes: As a result of this activity, the participant will be able (1) to identify three types of items that can be included in a linguistically fair assessment of syntax, and (2) to identify key concepts important to the development of the syntax items on the proposed assessment.

Grasping grammar involves moving to an abstract level that is so natural we do not realize how far it is from common sense. Its naturalness flows from the fact that much of it is innate, like vision. Yet like vision, either big problems (complete blindness) or tiny ones (imperfections of focal length) can be present. The challenge of communication disorders is to identify the deeper factors that lie beyond superficial description. Our presentation does not focus on dialect variation, but rather on those features of English that are constant across dialects. They therefore offer the opportunity for dialect-neutral diagnosis of language disorder. We begin with some simple examples and move to the kinds of sentences that may cause children to face real challenges and may cause real failure.

A linguistically fair syntax assessment can include these item types: wh-questions,

Evaluating Language Variation: Distinguishing Dialect and Development from Disorder; Editors in Chief, Nancy HelmEstabrooks, Sc.D., and Nan Bernstein Ratner, Ed.D.; Guest Editors, Harry N. Seymour, Ph.D., and Barbara Zurer Pearson, Ph.D. Seminars in Speech and Language, volume 25, number 1, 2004. Address for correspondence and reprint requests: Thomas W. Roeper, Ph.D. Professor, Department of Linguistics, University of Massachusetts Amherst, Amherst, MA 01003. E-mail: roeper@linguist.mass.edu. ${ }^{1}$ Professor, Department of Linguistics, University of Massachusetts Amherst, Amherst, Massachusetts. Copyright (C) 2004 by Thieme Medical Publishers, Inc., 333 Seventh Avenue, New York, NY 10001, USA. Tel: +1(212) 584-4662. 0734-0478,p;2004,25,01,041,056,ftx,en;ss100182x. 
passives, and articles. Our focus falls on three key concepts in these domains:

1. Implicit grammatical relations: How do we grasp silent, elliptical knowledge buried in short sentences with much left out?

2. Core properties of questions: the rules and restrictions for syntactic movement within and across clauses and the requirement to answer exhaustively.

3. Discourse linking: How do we make connections across sentences?

These are among the major concepts that can be evaluated by the syntactic assessments we have been exploring, but they represent only a few of the grammatical concepts that a competent speaker must master. They involve many subtle features of everyday language, which are often the missed signals that make conversations go awry.

Following the section on key concepts, we explain the rationale for the items on our assessment, how they are constructed, and some of the specific research results that support their use. We finish with some suggestions about how to elaborate these concepts to help the children who fail on the items gain a better understanding of them.

\section{KEY CONCEPTS}

\section{Implicit Grammatical Relations: Ellipsis}

We often are unaware that much of what we think we say actually is not stated. If someone says, "Here are some berries. Do you want some?" and then someone asks what was said, one might reply "I asked if you want some berries." However, that was never actually said, only "Do you want some?" The hearer must know to fill in the missing "berries."

Parents' speech is constantly elliptical, even with very young children. Here is a dialogue with a 2-year-old child from the CHILDES database $^{1}$ :

\section{Mother: There isn't any tapioca.}

Child: Have milk.

Mother: There isn't any.

Mother: We'll make some this afternoon.

Child: (unintelligible) make some (unintelligible).
Both any and some could refer to an unspoken milk, but upon reflection, "make some" seems to refer to tapioca. The child (and we) must figure it out-and it looks like the child may be having trouble. These are elementary examples, but the possibility for confusion is clear.

\section{Ellipsis (Implicit Grammatical Relations) in Passive Sentences}

The need to reconstruct missing information also arises if I say:

1. The bike was stolen.

If someone asks what was said, one might say, "I just said that someone stole the bike." But actually, someone was never mentioned and we had to supply the missing agent of stolen. This may seem inevitable in a sentence such as (1), because there cannot be any stealing without someone to do it. But it is not inevitable in a contrast such as:

2. The apple dropped.

3. The apple was dropped.

Only in sentence (3) is a missing agent implied. Unlike steal, it is not the verb drop that demands an agent, but the passive construction $($ was $+e d)$. Moreover, the missing agent cannot be the same as the object. If I say:

4. John was being washed.

The grammar of the sentence implies that a missing agent is present who is someone other than John (even though John could wash himself). This is a property that we call "disjoint reference" and it means simply that the subject and object are different.

\section{Properties of Questions: Exhaustivity}

A second key concept involves what we have called the "bottomless nature" of question words and other words that involve "quantifiers." If someone asks, "What is in your living room?" we could answer with a single word: "furniture." But we could also enumerate every object, or even describe the air, the windows, the views, the people, or colors. Unless 
modified by context, questions in principle demand an answer that is full and exhaustive.

These criteria can be captured in the term exhaustive set. Imagine that you are in court and the lawyer asks, "Who was in the car the night of the murder?" If there were three people and you answer with just one, you are liable for perjury. The question requires that we exhaustively mention everyone in the set of people who were in the car.

Children may fail to grasp that a question calls for an exhaustive set answer. A competent speaker, regardless of dialect, needs to understand that the word who or what by itself carries the set interpretation.

\section{Properties of Questions: Movement}

A question achieves a focus by an operation called movement. In the following example, we move the question word what to the front:

\section{I see a man, a woman, and a horse. $\rightarrow$ What do I see (-)?}

We can do the same thing with a complex sentence and move what over two clauses:

Complex: What did she say she saw (-)?

Here we have bumped into one of the deepest properties of grammar: potential infinity. We could just keep on going:

What do you think I think the teacher said that Mary told John she saw (-)?

Here we have expanded the same question over five clauses, and it still refers to the object of see. It is precisely the ability to swallow effortlessly this kind of potential infinity that is the essence of language ability.

\section{Movement, Ambiguity, and Barriers to Movement}

There can be even greater complexity in these structures. The question word can be potentially infinitely far away from the position it started from, as with see above, and it can also be ambiguous. In the following sentence there are two different possible when sequences:

\section{When did she think (-) \\ she lost her purse (-)?}

We could be asking when she said it or when she lost it. This can, of course, be an infinitely repeatable ambiguity as well:

$$
\begin{gathered}
\text { When did you say (-) she thought (-) she lost } \\
\text { it (-)? }
\end{gathered}
$$

It may appear as if we are complicating the situation and not the syntax, but it becomes clear that these ambiguities are regulated by syntax when we insert another question word:

When did you say (-) bow she thought she lost it?

A bit of reflection reveals that now all of the "lower" when positions are completely cut off and the question refers only to when the saying was done. One question word cannot jump over another. The second question word is called a "barrier."

Although such concocted sentences may seem strange to the reader, they are the stuff of daily life for children. Parents and children use these embeddings readily. One can easily imagine a 5-year-old child saying a sentence to a sibling that has three embedded clauses in it:

Mom said you said I did it, but I didn't.

\section{Articles and Discourse Linking}

Finally, we need an intuitive grasp of what articles do. If I say:

John bought a hat. The hat was green.

The the tells us that the bought hat and the green hat are the same. Once we introduce a noun with an indefinite article $(a)$, we can refer back to it with a definite article (the). Again, this is far from inevitable. Many languages have no articles and so the connection has to be made differently.

One might think that common sense tells us the connection is there. But, actually, that kind of common sense easily wanders into 
significant error. Compare the following two sequences:

I bought some cats. The cats are strange. I bought some cats. Cats are strange.

Without the, we refer to cats in general; with the, we refer to exactly the cats we bought, (who might be unusual in being strange).

The same connection can be achieved via our knowledge of the world. We can use a definite article to refer to a part of something already mentioned, even though the part itself has not been mentioned. We can say:

Take a cup. Use the handle.

In this context, we mean "the handle of the cup." If we do not know the object, we can still use the to assume a part/whole relation. If I say:

John has a motor. The brinch is broken.

From these sentences, we are led by the article to assume that "the brinch" is a part of the motor. This, again, is the sort of knowledge that a competent speaker must have, but is not taught (see Schafer and deVilliers ${ }^{2}$ for a systematic review).

\section{CONSTRUCTING A DIALECT- SENSITIVE SYNTACTIC PROBE}

\section{Wh-Questions}

The wh-question comprehension items of the syntax probe are of three types: double whquestions, embedded clauses (with false complements), and barrier questions. The double wh-questions involve exhaustivity, the embedded clause questions involve knowledge of implicit relationships and movement of the question word from the position of the constituent it replaces, and the barrier questions involve constraints on movement.

\section{DOUBLE-WH: TWO QUESTIONS, TWO EXHAUSTIVE SETS}

It is possible to ask two questions at once. With three words,

Who bought what? we utter a sentence that calls for two answers. The answers each require a set and seek exhaustivity. In addition, a third property must be honored: pairing. The question is answered as a paired list:

He bought fruit and she bought vegetables.

Questions of this kind require reference to all the members in the two sets in an ordered relation:

Person 1 bought Thing 1,

Person 2 bought Thing 2 .

Any questions can be used, for instance:

How did she catch what?

Our exploratory test (See Acknowledgments) investigated children's performance on this kind of question with stories of this type that make each pair somehow dramatic or interesting. One example constructed to test this concept might read: "This girl caught different things in different ways. She caught that crab with a net and the fish with her fishing pole. (pause) How did the girl catch what?"

\section{RESULTS: TYPES AND AMOUNTS}

We obtained three types of typical answers to such questions

1. Paired, exhaustive responses (correct):

- "She caught the crab with a net and the fish with a pole."

2. Singletons (incorrect):

- One element: "a crab" "with her pole"

- Both direct objects, no instruments: "crab and fish"

- One pair: "the crab with a net."

3. Other:

- "She fished a lot." "She was playing."

To see how revealing these questions are, we will provide a general overview of results from the field testing.

The difference between typically developing (TD) and language-impaired (LI) children shown in Figure 1 is statistically significant. In the analyses of variance, the main effects of development (age) and disorder (clinical 


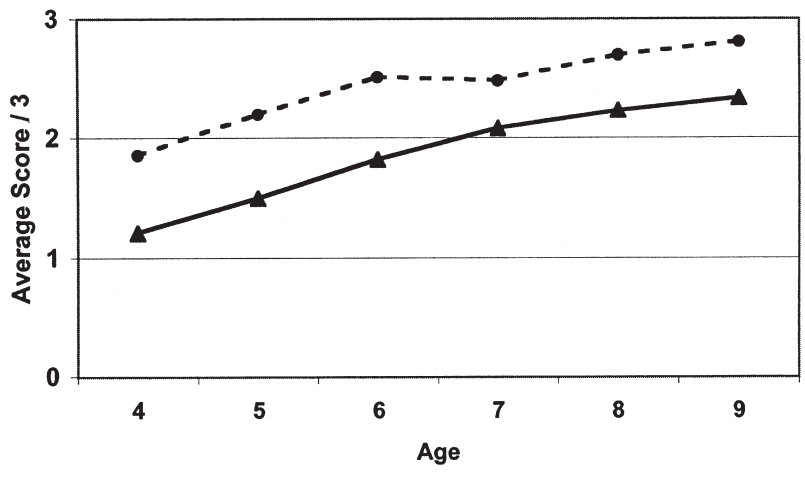

$\longrightarrow$ - Impaired - $\rightarrow$ - - Typical

Figure 1 Double wh-questions by Clinical Status.

status) are large and consistent [Age, $F(5$, $1002)=18.376, \quad p<.0001 ;$ Clinical Status, $F(1,1002)=49.966, p<.0001 ;$ Age by Clinical Status, $F(5,1002)=.449$, not significant]. We see that typically developing children can give appropriate answers two thirds of the time at the age of 4; disordered children give an appropriate answer one third of the time and remain consistently behind through the age of 9 .

Figure 2 shows that African American English (AAE) and Mainstream American English (MAE) speakers treat these structures essentially the same $[$ Age, $F(5,1002)=16.145$, $p<.0001$; Dialect, $F(1,1002)=6.435, p=.01$; Age by Dialect, $F(5,1002)=1.061, p=.381]$. Although the difference between the dialects was statistically significant, the simple effect at each age was not reliable except for ages 7 and 9, for which the number of subjects was very small.

Thus, these rare sentences achieve our goal: they produce a method to recognize and iden- tify a disorder without the diagnosis being affected by dialect in a critical age range. In addition, of course, they identify a disorder that has hitherto not been seen: an inability to handle a double question. The inability to handle the double question is pertinent to all questions because it forces out an important feature of questions: the reference to a potentially infinite set. The children who cannot answer double questions will be those who inappropriately give a single response to simple questions in ordinary life, such as the child who mentions just one ingredient when you ask, "What do you need to make a cake?"

\section{CASE STUDIES}

Here are two example children from our field testing who give different responses to double wh-questions:

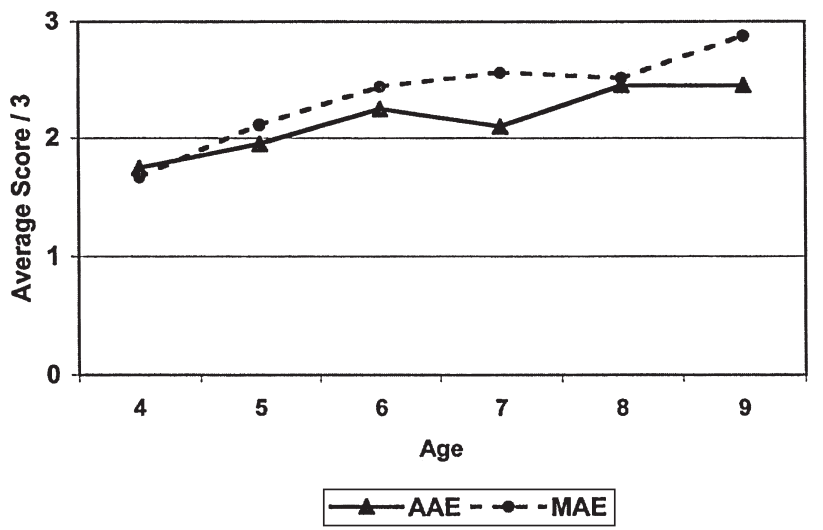

Figure 2 Double wh-questions by Dialect. 
Child A

\section{Child B}

banana and a apple The Mom ate the apple and the Dad ate the "nana

fish and crab fish with her pole, crab with a net

Only child B sees the requirement of a paired answer.

\section{HIDDEN FALSE-CLAUSE QUESTIONS}

Complex sentences make substantial cognitive and semantic demands, and require the child to understand implicit relationships across clauses. When one clause is inside another clause, the notion of the truth of the sentence must refer to the whole, not the parts. Thus, if I say,

Who did John say stole the bread?

I may not have said who actually stole the bread. If a child were to treat such a sentence as a conjunction, then both parts would require true answers-we would really be asking who did it, not just who John said did it:

Who did John say and (who) stole the bread?

The child must see that putting one verb inside the other changes the truth value: Now we do not have to know or say who stole the bread, just who John said did it. Children definitely can stumble over this computation.

When we answer an embedded question like this, our answer must address the verbs from both clauses: who John say-steal. If our answer is based on just one of the verbs (e.g., who stole?), we show that we have not understood the relationship of the embedding.

Here is how we presented this kind of question in our field testing:

This girl snuck out one night when her little sister was asleep and brought a pretty plant as a surprise. The next day the little sister saw the bag from the store and asked, "What did you bring me?" The older girl wanted to keep the surprise until later so she said, "Just some soap." (pause) What did the girl say she brought?

Here are typical answers to false-clause questions:
1. "Long-distance" two-clause responses (correct):

- She said she brought soap (she say-buy)

2. One-clause responses (incorrect):

- (She bought) a pretty plant.

3. Other:

- a bag, I don't know.

If we look at answers to this type of question from the field testing, we find again that there is a strong effect of development, but almost no difference in AAE/MAE performance [Age, $F(5,1002)=11.789, p<.0001$; Dialect, $F(1,1002)=.978, p=.323$; Age by Dialect, $F(5,1002)=1.471, p=.197]$. TD and LI groups are significantly different [Clinical Status, $F(1,1002)=5.866 ; p=.016]$. We find again that children who have disorders are systematically less likely to give the correct answer:

Here are the same two example children as above:
Child A

a plant
Child B

some soap
Child A has apparently either completely ignored the verb say or imposed an independent constraint that the lower clause must be true.

\section{BARRIER QUESTIONS}

These questions require the child to understand the concept of movement; they must know which clause a wh-word has moved from to answer correctly. When there is more than one clause and one or more question words, the child must be able to figure out with which verb each question word is associated. They need to be able to tell when the question is ambiguous (and there is more than one correct answer, such as, "When did she say she lost her purse?") and when there is a barrier to movement which restricts how elements can move and limits the "when" question to one correct answer (e.g., "When did she say how she lost her purse?")

In answering these questions, children sometimes make a very important kind of error. It can appear to be a kind of arbitrary misunderstanding, but in fact it is very systematic, 
persists for a long time, and resembles grammatical structures in other languages. Such sentences are those that involve two question words, but only one question; the second question is known as an "indirect question" that we do not usually answer:

Why did you say who you saw?

This sentence asks only about why you said something, not about who you saw or why you saw them. Many languages around the world allow a construction where you answer the question in the middle (e.g., the "medial" question) and the first wh-word is just a clue that another word is following. It is as if one said:

What did you say how you were going to swim?

where we understand that we are to answer "how you swim." In most cases, English does not allow the possibility of answering the medial question.

De Villiers and Roeper ${ }^{3}$ explored these questions and responses in extensive experimentation in six languages. Here is a story from their research that we presented to children in our pilot studies:

This boy was climbing in the forest one afternoon when he slipped and fell out of the tree. That night when he was taking a bath, he saw that he had bruise on his arm and he called to his dad to tell him that he had hurt himself.

When did he say how he hurt himself?
Here are typical answers:

1. Short-distance responses (correct):

- (When did he say...?) in the evening in the bath.

2. Medial answers (incorrect)

- (... how fell?) he slipped from the tree

3. Long-distance responses (incorrect)

- (When ... fall?) in the afternoon "when he was climbing"

4. Other

- "I don't know."

Our two case studies follow the same pattern as before:

\section{Child A}

from the tree

\section{Child B}

when he was takin' a bath

Again we find in Figure 3 that disordered children consistently do worse on these constructions across the age range, but there is no interaction between the factors [Age, $F(5$, $1002)=42.110, \quad p<.0001$; Clinical Status, $F(1,1002)=85.857$; $p<.0001$; Age by Clinical Status, $F(5,1002)=1.265, p=.277]$.

Note that what we call the medial response persists among TD children well into the 7- to 8 -year range. Its persistence must have something to do with the fact that it is quite acceptable in Russian (with how) and German (with what). Thus, this interpretation is possible in some languages but not English. (The interested reader can find extensive discussion of such cases in the acquisition literature. ${ }^{3,4}$ [Seigmuller and Weissenborn, unpublished report])

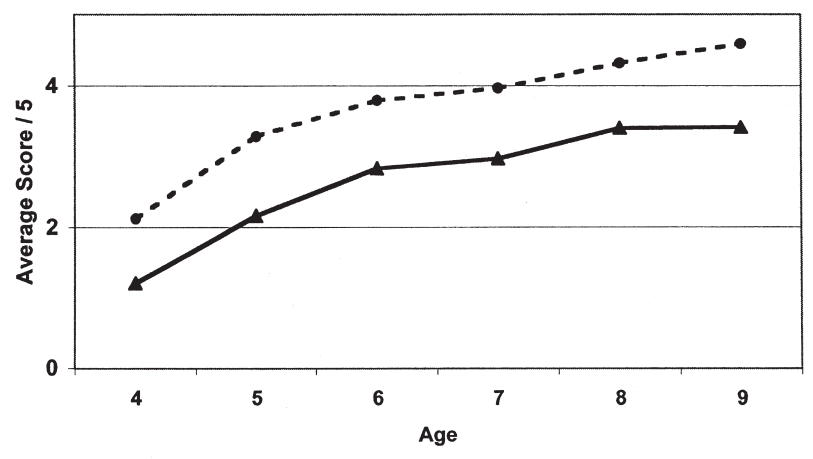

Figure 3 Barrier questions by Clinical Status. 


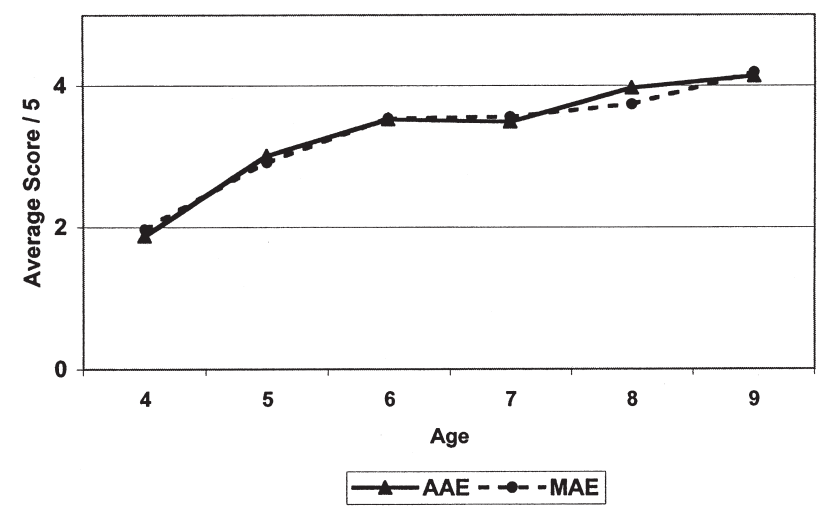

Figure 4 Barrier questions by Dialect.

As shown in Figure 4, we find in these items a strong age effect but unlike clinical status, dialect has virtually no impact [Age, $F(5,1002)=42.468, p<.0001$; Dialect, $F(1,1002)=.073, p=.787$; Age by Dialect, $F(5,1002)=.494, p=.781]$. This pattern makes barrier questions ideal for a linguistically fair syntax assessment.

\section{Passive Subdomain}

We turn now to another construction where some information is totally unspoken but still known to any competent speaker: passive. The passive comprehension items test children's understanding of movement and also implicit relationships; that is, hidden information that is implied by the grammar of the sentence, but not stated in words.

The most salient feature of passive constructions bears an important relation to whquestions: movement is involved. The object moves to the subject position. Other properties, however, as we have outlined above, play an important role in a sentence such as:

The plant was dropped.

There is an implied and disjoint agent (that is, someone dropped the plant and the agent is different from the subject).

\section{MOVEMENT IN PASSIVES}

Control of this construction is shown if the child can reliably discern the subject and the object (Fig. 5). For example,
The dog was pulled.

The response pattern to questions of this type (Fig. 6) suggests that both LI and TD children do not always understand this distinction (as other experiments have shown as well), but the statistical comparison shows there to be a TD/LI gap [Age, $F(5,1002)=43.526$, $p<.0001 ;$ Clinical Status, $F(1,1002)=$ 33.949; $p<.0001$; Age by Clinical Status, $F(5,1002)=.719, p=.609]$. A separate analysis of variance showed Age and Age by Dialect effects of similar magnitude and confirmed that there was no reliable AAE/MAE difference in performance [Dialect, $F(1,1002)=2.686$; $p=.102]$.

However, the ability to pass this item type does not demonstrate that children comprehend how the passive carries knowledge of an implicit, disjoint agent.

\section{DISJOINT, IMPLICIT AGENT}

The following illustration (Fig. 7), which is similar to those on the comprehensive language test, provides a choice between an active and an passive with a disjoint agent:

The bear was being washed.

If children prefer a result-passive reading, they do not have to assume a disjoint agent because a result-passive is much like an adjective that can be formed without any movement at all:

The bear was washed.

Compare: The bear was brown. 


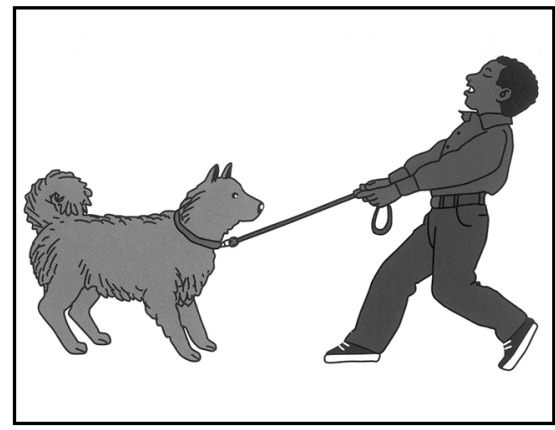

A

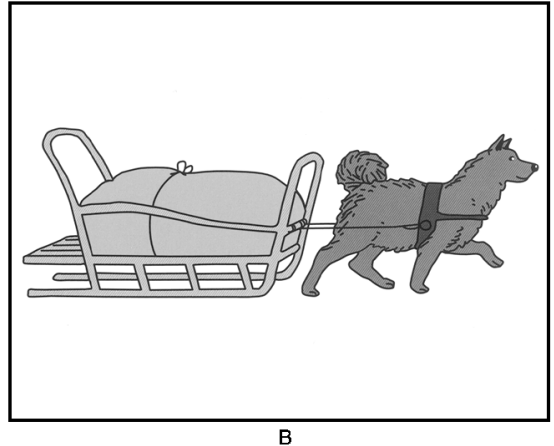

B

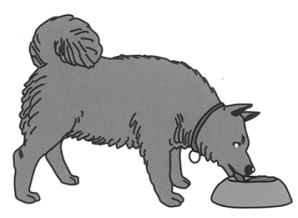

Figure 5 Basic passive item. (Show me: The dog was pulled.)

Therefore, this extra complication is crucial to the determination of whether the child understands the sentence to be a passive at all.

Again, the familiar pattern is present (Fig. 8), although comprehension comes somewhat later than we saw with the simple passives. The statistical comparison shows there to be a TD/LI gap with a reliable interaction by age [Age, $F(5,1002)=28.708, p<.0001$; Clinical Status, $F(1,1002)=29.788 ; p<.0001$; Age by Clinical Status, $F(5,1002)=3.935, p=.002]$.
A separate analysis of variance showed Age and Age by Dialect effects of similar magnitude and no reliable AAE/MAE difference in performance (Dialect, $F(1,1002)=3.317$; $p=.07)$.

\section{DISTINGUISHING THE AGENT BY-PHRASE FROM A LOCATION BY-PHRASE ("BY THE TREE" AS IN "NEAR")}

The special role of the $b y$-phrase as an agent carrier in passive can be isolated through

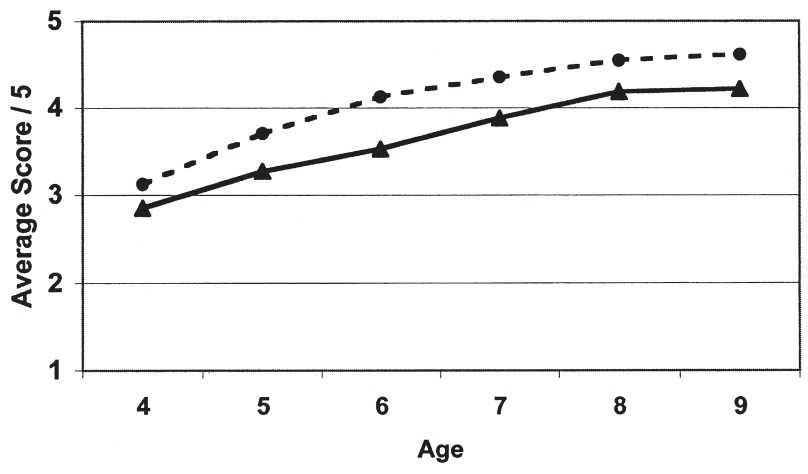

$\longrightarrow$ Impaired - $\rightarrow-$ - Typical

Figure 6 Basic passives by Clinical Status. 

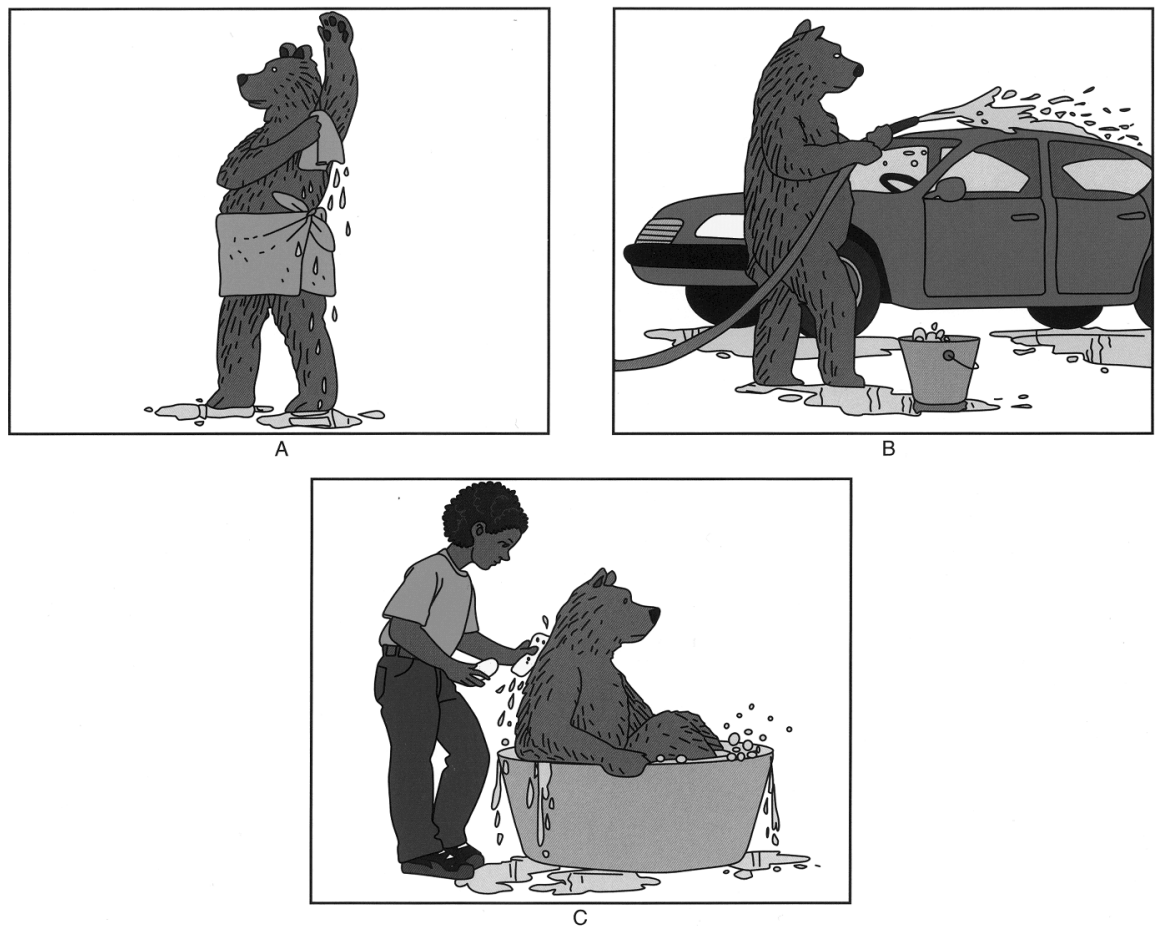

Figure 7 Complex passive item 17. (Show me: The bear was being washed.)

sentences that block this function. When a progressive sentence is used, the $b y$-phrase can only be a locative, not an agent. Although no linguistic theory can explain very well the absence of an agent reading, it is still useful to us as a diagnostic because some children fail to recognize this:

1. The branch was dropped by the man (passive/agent).

2. The branch was dropping by the man (locative phase).
We find here that there is a significant challenge to LI children and some delay even among TD children. The statistical comparison shows no AAE/MAE difference, but a reliable difference between TD and LI children [Age, $F(5,1002)=9.275, p<.0001 ;$ Dialect, $F(1$, $1002)=.043 ; p=.835 ;$ Age by Dialect, $F(5$, $1002)=1.681, p=.136]$. A separate analysis of variance confirmed Age and Age by Clinical Status effects of similar magnitude, and a strong TD/LI difference in performance (Clinical Status, $F(1,1002)=10.122 ; p=.002)$.

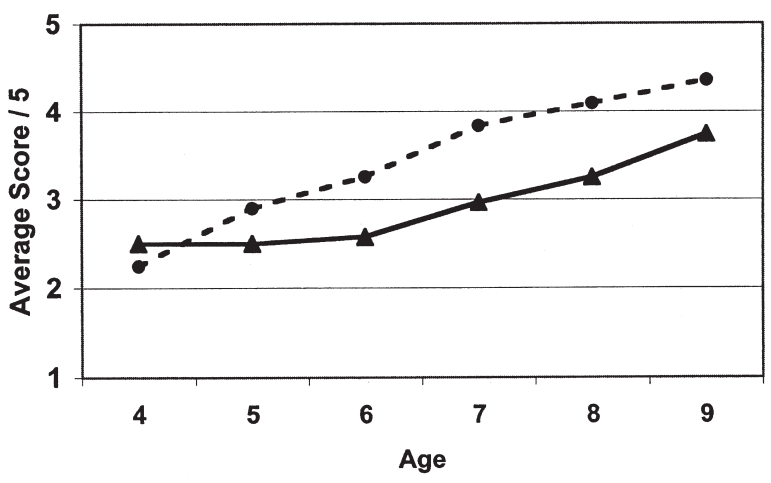

$\longrightarrow-$ Impaired - $\rightarrow--$ Typical

Figure 8 Complex passive items by Clinical Status. 
If we now return to our two case studies, we find again that they pattern similarly:

Child A fails the simple passives 2 of 4 times, fails the complex passive 3 out of 4 times, and allowed an agent reading for a locative by-phrase.

Child B (18221) succeeded with simple passives 3 of 4 times and with the complex passives 2 of 4 times. Both locative by-phrases were correct.

\section{Articles Subdomain: Understanding the Principles of Discourse Linking}

Articles are among the most automatic forms of speech English speakers have. Yet, articles make subtle demands on children's syntax and semantics; they engage their knowledge of context and presupposition. Consider that articles constitute one of the most vexing barriers to perfect English that Asian speakers encounter. We say, "I got a cat" and are not tempted to say, "I got cat." The simple explanation for this problem is that Asian languages have no articles. The deeper question of why articles are so difficult to explain is still unclear.

\section{Some of the Basic Uses of Articles that Can Be Tested with Children this Age}

\section{ARTICLES: DEFINITE VERSUS INDEFINITE}

The most elementary bridge between sentences is simply filling in missing information, as we indicated above. We begin with the same kind of discourse link between noun phrases:

John has some berries.

I would like some (berries).

I would like the berries.

Shifting some to the depends on the recognition that it is the same berries. So also can "a dog" shift to "the dog" once it has been mentioned.

The same link is present when we apply common knowledge to justify the presupposed familiarity that the implies. That is, if we say:

John has some berries. The stems are still on. (the stems $=$ the stems of the berries)
We must still reconstruct a hidden connection to the berries, which again justifies the. It is an interesting fact observed by Schafer and deVilliers ${ }^{2}$ that this part/whole connection is easily available to children in comprehension.

Indefinite articles present their own challenges. They may be either specific or nonspecific. A sentence such as,

I want to buy a book.

is ambiguous between a reading where there is a specific book that one wants and the general desire to buy a book.

Another use of the specific is when an item is known to the speaker but not the listener, which means the whole communicative situation must be conceived of. I can say,

I have a picture at home.

If I were in the room, I might get a the ("Look at the picture on the wall!"). However, because the listener does not know what picture is being referred to, we must use $a$.

In sum, the English article system is especially complex and, not surprisingly, it is not learned all at once by TD children. In most respects, the article system is learned alike in both MAE and AAE.

\section{EXAMPLES OF POTENTIAL ITEMS TO ASSESS ARTICLE KNOWLEDGE}

Fortunately, despite the complexity of the phenomenon, the method of eliciting answers has proven splendidly simple. We tried to avoid the concreteness that is delivered by pictures or toys, which confounds the very phenomena in which we are interested. ${ }^{2,5}$ All we do is ask children to finish a story where the part/whole definite or other indefinites are implied.

Part/whole story:

Jack wanted to eat a coconut, but first he had to take something off it. What did he take off it?

(The peel, the shell, the brown thing)

Familiar-the story:

A snake and a bird were sitting on a rock.

They were friends. One of them flew away.

Guess which. (The bird) 
Specific- $a$ story:

I'll bet you have something sharp in your kitchen at home. What is it? ( $A$ fork, $a$ knife)

Nonspecific- $a$ story:

Chuck wants to take his teddy bear, his favorite game, and his baseball to school for show-and-tell.

What can he put them all in? ( $A$ bag)

\section{TYPES OF ARTICLE ERRORS}

Although it is commonly assumed that children prefer specifics, we find that they substitute indefinite $a$ for the 8 times more often than they substitute the for $a$. This may seem surprising from a common sense cognitive perspective, but it follows naturally from a grammatical perspective. The unmarked noun has the most abstract meaning: "John likes cake." An indefinite provides minimal marking (a cake), and the definite carries the most information (I like the/that cake).

The pattern of correct article use in TD and LI children is evident in Figure 9 [Age, $F(5,1002)=52.874, p<.0001$; Clinical Status, $F(1,1002)=59.893 ; p<.0001 ;$ Age by Clinical Status, $F(5,1002)=6.959, p=.02]$.

As we saw in the article by Pearson, ${ }^{6}$ again there was virtually no difference across dialects (Fig. 10) — an ideal outcome in selecting culturally and linguistically fair items.

\section{Case Studies Revealed}

It is clear that Child A has shown worse performance on wh-questions, paired readings, and passive. Now we can ask an important question: Who are these children? Their backgrounds are described below.

Child A is a 5-year-old white female from the South whose parents have a high school education. She is an MAE speaker and is not receiving speech or language services.

Child B is a 4-year-old African American boy from the Midwest whose parents have a high school education. He speaks with "Some Variation from MAE" (see Ciolli and Seymour ${ }^{7}$ ) and is not receiving speech or language services.

These case studies then provide us with an ideal demonstration of the fact that deep problems can exist for MAE speakers that may not be problems for a dialect speaker. Our statistical results are borne out with individuals.

\section{SUGGESTIONS TO HELP CHILDREN LEARN THESE KEY CONCEPTS}

These examples all circle around questions where movement, sets, and truth values are written into the structure of language in ways that go beyond cognitive ability. What does this say about possible remediation? Initially, one can discuss stories of the kind used in the test with children and engage them in a deeper appreciation of them.

It will help children understand better if we take away the ambiguities that are carefully built into the test and instead create contexts that support the hidden meanings and make them evident. Some simple suggestions that address each of the missing properties follow.

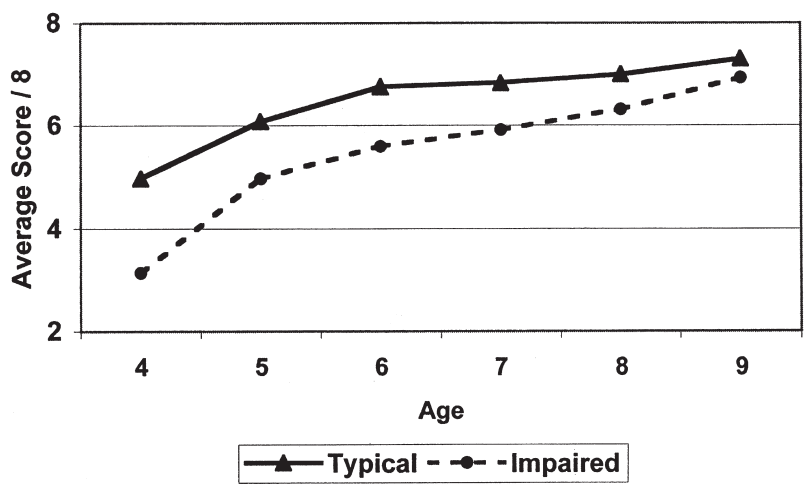

Figure 9 Article scores by Clinical Status. 


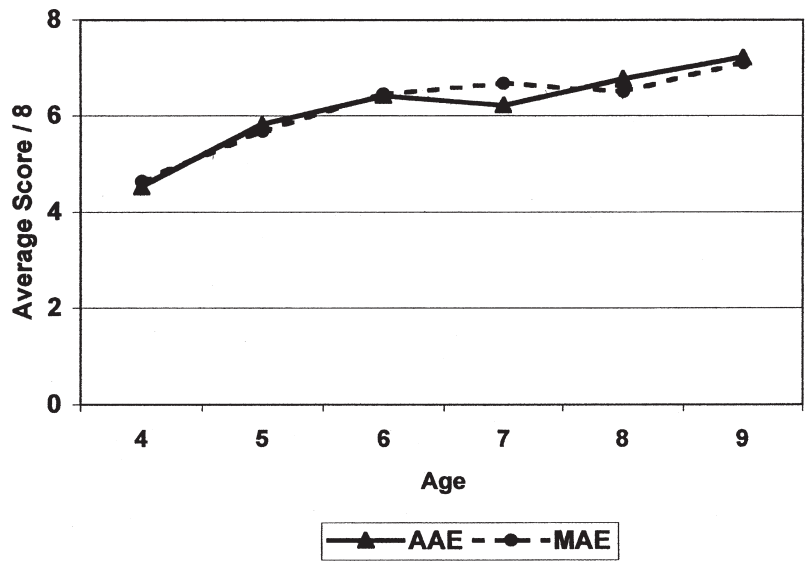

Figure 10 Article scores by Dialect.

They are all contextually clear and yet inherently indirect efforts to bring about a grammatical realization in a child.

\section{Movement}

If a child does not understand movement, we can illustrate movement first with sentences in which content is not being questioned, and so nothing needs to be supplied by the child. Thus if we say:

$$
\text { Milk John likes. }
$$

and then ask:

\section{What does John like?}

we have given the crucial information, not in object position at the end, but already focused in topic position at the front of the sentence.

\section{Sets}

For the child who does not understand the need to answer with a set, we can imply the multiple nature of wh-words through other lexical and contextual support. We can make it so that it would not make sense to answer a wh-question as if "who" meant "who-somebody" and not "who-everybody."

If we ask:

Who plays together?

Who shared the ice cream?

Who helped each other? these sentences are difficult to answer sensibly with a single person. In contrast, we can imagine situations for which a single answer is impossible: For example, we can present a scene in which 20 people are lifting a rock and ask:

\section{Who lifted the rock?}

In such as example, it would not really be correct to point to just one person.

\section{PAIRING AND EXHAUSITIVITY}

To help children grasp the concept of paired exhaustive answers, one might reverse the conversational thrust to focus on exhaustivity. Suppose we have three people painting three houses and we say only:

John painted the big house.

and then ask:

Did I tell you which person painted which house?

The answer should be "no" because you only mentioned only one person and only one house. The "no" shows that the question really requires an exhaustive, paired response. In addition, one could ask the child to finish the description.

\section{PASSIVE IMPLICIT AGENT}

To help a child who has difficulty understanding the passive, the natural step is to make the hidden information explicit. First, one would 
want to articulate the presence of unspecified subjects:

Someone dropped the bowl.

Second one would want to use a passive in the same situation with an explicit agent:

The bowl was dropped by someone.

Finally, one can reiterate the agentless form:

The bowl was dropped.

Thus, one could recreate the steps hidden in the formation of the passive for the child. One can show which sentences with full information are equivalent to a sentence with hidden information.

\section{ARTICLES}

One method to bring children to the awareness of part/whole relations could be to embed the relation in a conversation in which it is used correctly and see if they agree. If we show the child a picture with two cats in it, one with a brown tail and one with a red tail, and say:

Here's a cat with a long tail that is brown.

The tail is not red.

Is that right?

If the child says "yes," even though there is a red-tailed cat in the picture, then the right connection to the tail was clear. But if the child says "no," then we need to say that "the tail" picks out one particular tail and not any tail.

\section{CONCLUSIONS}

We have shown that the assessment of complex aspects of children's syntactic development between the ages of 4 and 9 can be carried out in a dialect-neutral fashion. Such assessments provide the clinician with a substantial profile of the child's language strengths and weaknesses, not just a diagnostic categorization. We have isolated certain grammatical constructions in sufficient depth that one can treat each one as a form of independent appraisal. Like ear, nose, and throat medicine, the problems in these constructions can be either connected or independent. The dialect-neutral testing allows one to achieve both a composite picture of abilities and an individualized analysis.

Our approach has allowed us to tap deep grammatical principles through unusually short and simple sentences. This allows us to minimize the impact of extraneous world knowledge, problems of parsing, or memory. We have been able to do this by using the crucial kinds of examples that have played a role in modern linguistic theory.

These materials and procedures focus on the development of aspects of language that are vital for success in early schooling and the transition to literacy. School is full of intricate questions, dialogues with missing information, and unusual connections between sentences that articles help to convey.

\section{ACKNOWLEDGMENTS}

This work was funded in part by National Institutes of Health (NIDCD) under Contract \# N01 DC8-2104 and Grant \# R01 DC 0217104 to Harry Seymour, Principal Investigator, at the University of Massachusetts Amherst, with Thomas Roeper and Jill de Villiers at the University of Massachusetts and Smith College, as co-investigators. It was accomplished in conjunction with The Psychological Corporation of Harcourt Assessment, Inc., San Antonio, TX.

The tests that are the products of this research collaboration are the Diagnostic Evaluation of Language Variation (DELV) assessments, the DELV Screening Test, DELV Criterion-Referenced edition, and the DELV Norm-Referenced edition. The phrase "evaluating language variation" refers generally to the assessment processes discussed in this issue. The term $D E L V$ is the name trademarked by The Psychological Corporation of Harcourt Assessments, Inc., and refers to the specific tests that are the outcome of the extensive research described in this article. The specific tests are referred to as the $D E L V-S T$, or "screener," or the DELV-CR, DELV-NR, or the "full diagnostic test," as appropriate. Questions about the principles underlying the tests can be referred to the authors of this issue (Seymour, Roeper, de Villiers, de Villiers, Pearson, and Ciolli). Questions about the tests 
themselves should be addressed to the Project Leader at The Psychological Corporation of Harcourt Assessment, Inc.; Lois Ciolli, Senior Research Director.

\section{REFERENCES}

1. MacWhinney B. The CHILDES (Child Language Data Exchange System) Project: Tools for Analyzing Talk, 3rd ed. Mahwah, NJ: Lawrence Erlbaum; 2000

2. Schafer RJ, de Villiers J. Imagining articles: What 'a' and 'the' can tell us about the emergence of DP. In: Howell SC, Fish SA, Keith-Lucas T, eds. BUCLD 24: Proceedings of the 24th Annual Boston University Conference on Language Development, Vol. 2. Boston, MA: Cascadilla Press; 2000:609-620

3. Roeper T, de Villiers JG. Lexical links in the whchain. In: Lust B, Hermon G, Kornfilt J, eds.
Syntactic theory and First Language Acquisition: Cross-Linguistic Perspectives. Vol. II: Binding, Dependencies, and Learnability. Hillsdale, NJ: Lawrence Erlbaum; 1994:357-390

4. Weissenborn J, Roeper T, De Villiers J. The acquisition of wh-movement in German and French. In: Maxfield TL, Plunkett B, eds. Papers in the Acquisition of WH. Proceedings of the University of Massachusetts Roundtable UMOP Special Edition; 1990:43-73

5. Maratsos MP. The Use of Definite and Indefinite Reference in Young Children: An Experimental Study of Semantic Acquisition. New York: Cambridge University Press; 1976

6. Pearson BZ. Theoretical and empirical bases for dialect-neutral language assessment: contributions from theoretical and applied linguistics to communication disorders. Semin Speech Lang 2004; 25: $x x x-x x x$

7. Ciolli L, Seymour HN. Dialect identification versus evaluation of risk in language screening. Semin Speech Lang 2004;25:33-40 
